\title{
Research Paper \\ Development and Implementation of Discharge Planning Service in Roozbeh Hospital
}

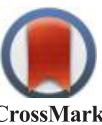

\author{
Hamidreza Toufighi ${ }^{1}$, Vandad Sharifi' ${ }^{2}$ Javad Alaghband-Rad ${ }^{3},{ }^{*}$ Behrang Shadloo $^{4}$
}

1. Resident of Psychiatry, Psychiatry and Psychology Research Center, Tehran University of Medical Sciences, Tehran, Iran.

2. Psychiatrist, Associate Professor, Department of Psychiatry, Psychiatry and Psychology Research Center, Tehran University of Medical Sciences, Tehran, Iran.

3. Child and Adolescents Psychiatrist, Associate Professor, Psychiatry and Psychology Research Center, Tehran University of Medical Sciences, Tehran, Iran.

4. Psychiatrist, Assistant Professor, Department of Psychiatry, Iranian National Center for Addiction Studies, Tehran University of Medical Sciences, Tehran, Iran

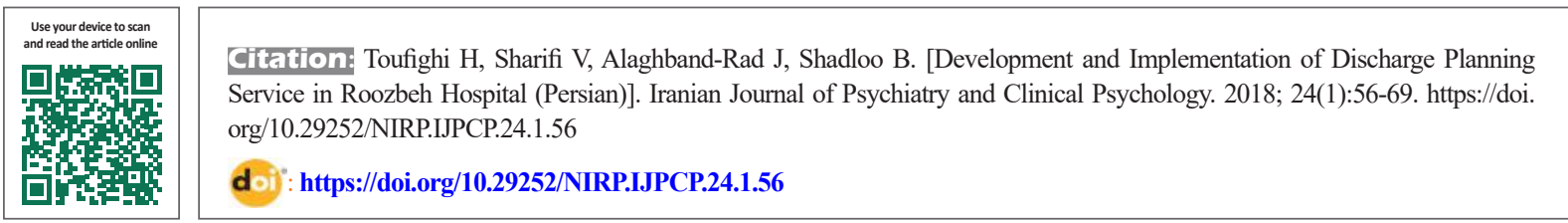

Received: 07 Nov. 2016 Accepted: 19 Jun. 2017

Key words:

Mental disorders,

Patient discharge, Health services

\section{A B S T R A C T}

Objectives Discharge Planning is a dynamic, collaborative and comprehensive process which is aimed to promote the continuity of care after discharge and providing necessary services and support to the client and his/her caregivers. Recently, the efficacy of discharge planning has been shown in multiple studies on factors like re-admission, treatment adherence, length of stay, treatment costs, symptoms resolution and patients' quality of life.

Methods We used an evidence-based service development model which contained five steps, including needs assessment, situation analysis, cost-effectiveness evaluation, prioritization and structural design. Results Needs assessment studies and situation analysis showed that Roozbeh Hospital had some deficits in adherence to outpatient visit and length of stay indices. In the next step, prioritization among possible interventions and designing the structure and details of this service in Roozbeh Hospital performed based on the principles mentioned in the literature, needs of the center and feasibility of services, and finally a comprehensive, collaborative and continuous program designed which includes some steps which are patient data registration, needs assessment, coordinating services and checklists of the affairs. Conclusion Development and implementation of the discharge planning service has been performed as the first one in Iran, in Roozbeh Hospital and requires to be evaluated and revised continuously, and we hope that this program be prominent to other psychiatric inpatient centers and also higher order mental health organizations.

\section{Extended Abstract}

\section{Introduction}

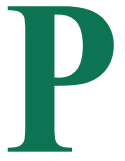

sychiatric disorders have a high prevalence and impose a huge burden to societies all over the world. Despite the recent trend to develop community-based psychiatry services, hospital-based services are still an inevitable part of treatment process, especially for patients in acute phases. Nowadays, interventions focusing on the transition phase from inpatient to outpatient are gaining increasing attention. Each patient, after discharge from psychiatric inpatient ward, needs to be monitored for optimum adherence to outpatient pharmacological and non-pharmacological services. Discharge planning is a dynamic, collaborative and comprehensive process, which is aimed to promote the continuity of care after discharge and providing necessary services and support to the client and his/her caregivers. Recently, the efficacy of discharge planning has been shown in multiple studies on factors like re-admission, treatment adherence, length of stay, treatment costs, symptoms reso-

* Corresponding Author:

Behrang Shadloo, MD

Address: Department of Psychiatry, Iranian National Center for Addiction Studies, Tehran University of Medical Sciences, Tehran, Iran.

Tel: +98 (21) 55421155

E-mail: behrang.shadloo@gmail.com 
lution and patients' quality of life. In line with the above, we decided to develop and implement a discharge planning service in Roozbeh Hospital.

\section{Method}

We used an evidence-based service development model which contained five steps, including needs assessment, situation analysis, cost-effectiveness evaluation, prioritization and structural design. In the first step, the researchers must perform a comprehensive review of literature in the target field, to estimate the level of necessity of the service for their goal. The second step contains the assessment of the current level of the services provided in the field, based on some standard indices. Cost-Effectiveness study of the service comes after that, and in the fourth step the researcher must prioritize the possible suggested interventions based on indicators like the burden of disease, cost-effectiveness, feasibility and affordability.

At the end, developing the service structure, process and details would happen. This is worth to mention that some authors refer to the fourth and fifth steps together as the implementation phase. Implementation is also defined as a series of specific activities directed to make a program with specific features work. This process contains determining the criteria for staff selection and education, providing pre-requisites, forms and devices, determining the data registry system, providing educational material, and choosing the corresponded and coordinator individuals for each step. The review of literature has been performed using international scientific databases including Medline, Elsevier and Cochrane; and domestic databases including Noormags, Magiran, SID and Medlib. The search performed on keywords including discharge planning, patient management, case management, service development, service implementation, and service planning, in English and Persian, with no time or study design restriction.

\section{Results}

In brief, the majority of references found the discharge planning service efficient on improving factors like re-admission rate, treatment adherence, length of stay, costs of the treatment, symptom resolution, quality of life, suicide rate, and patients' satisfaction. Needs assessment studies and situation analysis showed that Roozbeh Hospital had some deficits in both measured indicators. Based on the Health Information System of the hospital, we reviewed the adherence of the discharged patients to their outpatient visit, with more than half $(56 \%)$ of the patients had no outpatient follow-up visit after discharge, and $37 \%$ had come just one time. Although $44 \%$ of our patients showed some degrees of follow-up visit adherence and it is about the average (21$76 \%$ ) in other centers, this could be improved up to $47-95 \%$ like the centers developed discharge planning services. The other indicator was the length of stay, and its mean in Roozbeh Hospital (Based on the HIS data) was 28 days, and is above the national average (20.7 days), and far from other countries like Belgium (10.2 days), Italy (13.9 days) and Turkey (15 days).

In the next step, prioritization among possible interventions and designing the structure and details of this service in Roozbeh Hospital performed based on special needs of the center and feasibility of services, considering the principles mentioned in the literature such as continuity of care, availability of the service, comprehensiveness of domains, maximal patient and his/her family collaboration, and the patients' individuality. Finally, a discharge planning program designed which includes following steps: patient data registration, needs assessment, coordinating services and checklists of the services. Although the discharge planning is a team-based service which includes the psychiatrist, psychologist, social worker, nurse, day center staff, and surely the patient and his/her caregivers, every patient would have a specific contact-person called treatment mediator, whom is one of the nurses of the admission ward.

The content of discharge planning service in the time of admission ideally includes four sessions. The session zero contains welcoming the patient by the nurse and reminding the ward rules. The first session would be on needs assessment and planning for them, and the second session would be on specific educations for the patient and the caregivers. In the last session at the time of discharge, the forms and checklists will be fulfilled.

\section{Discussion}

Discharge planning service development is proven to be efficient in many ways, and even seems to be an ethical necessity. We had some limitations in our study such as lacking a good relationship with extra-organizational structures and social support systems, and having no prior experience in this field in the country. Meanwhile, the results of integration of the service in other services of the hospital would be gathered and published later. Development and implementation of the discharge planning service has been performed for the first time in Iran, in Roozbeh Hospital and requires to be evaluated and revised continuously, and we hope that this program be prominent to other psychiatric inpatient centers and also higher order mental health organizations. 


\section{Ethical Considerations}

\section{Funding}

This paper was extracted from the $\mathrm{PhD}$ dissertation of the first author in Psychiatry and Psychology Research Center, Tehran University of Medical Sciences.

\section{Conflict of Interest}

The authors declare no conflict of interest.

\section{Acknowledgements}

We wish to thank Dr. Zahra Mirsepasi, Dr. Valentin Artounian, Dr. Fatemeh Ranjbar-Kermani and the staff of Roozbeh Hospital day center for their invaluable contribution in our study. 


\title{
طراحى و ييادهسازى خدمت برنامهريزى ترخيص در بيمارستان روزبه
}

\author{
حميدرضا توفيقى'، ونداد شريفى"، جواد علاقبند راد"، "بهرنى شادلو"

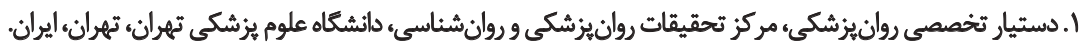

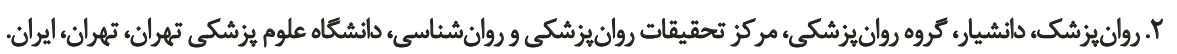

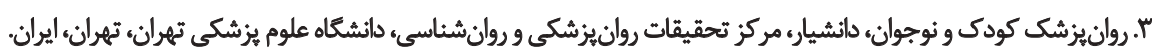

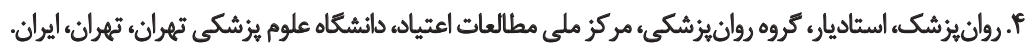

\begin{abstract}
حكيد

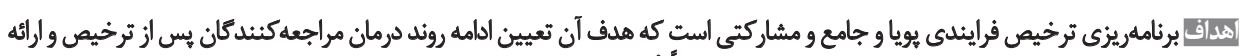

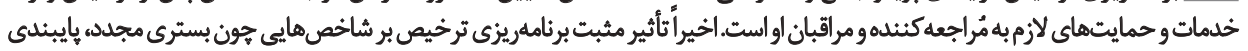

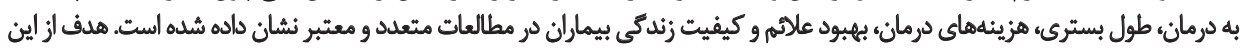

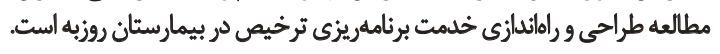

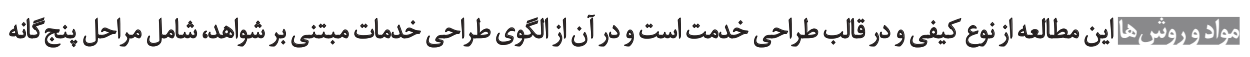

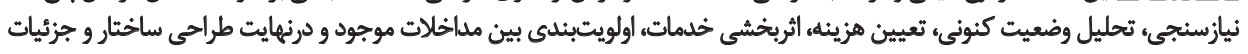
خدمت ملنظر استفاده شديه است كنيت

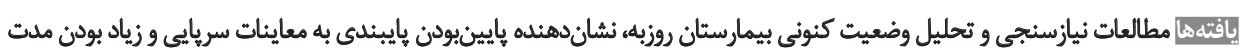

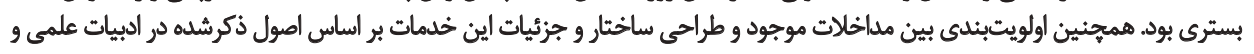

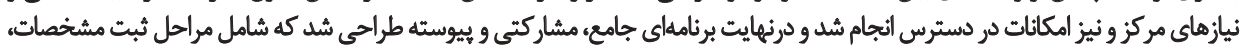

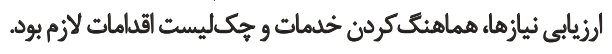

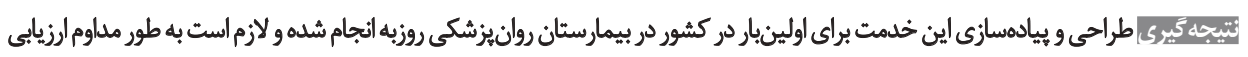

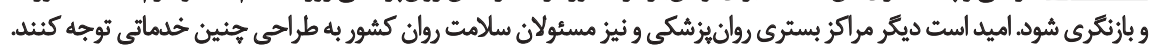

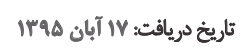

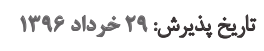
كليدوازوها:

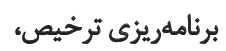 طراحى خدمثة، اختلالات روانيزشكى خلى

بيوستكى مراقبت، ' اهميت بسزايى در كيفيت خدمات دارد مقدمه

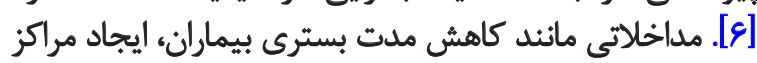

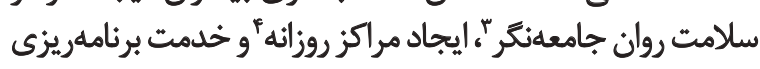

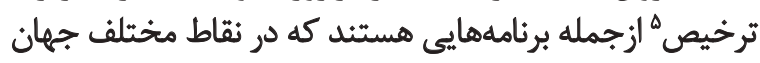

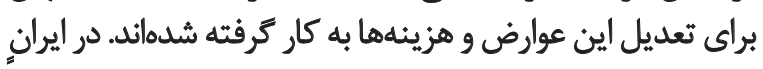

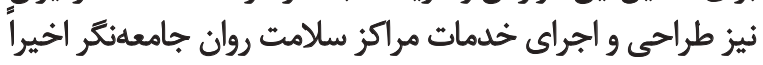

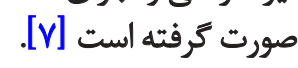

مراجعهكنيدكان به روانيزشك، بعد از ترخيص ممكن

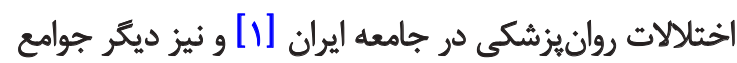

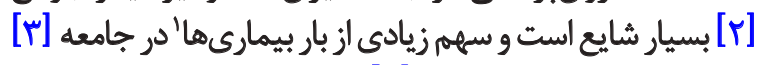

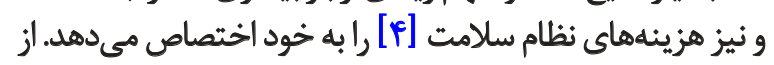

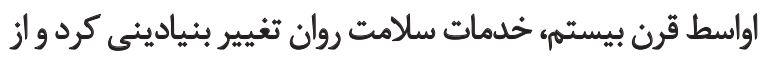

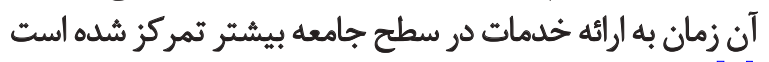

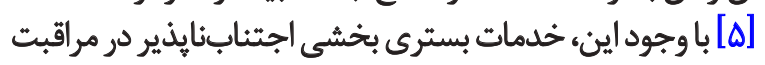

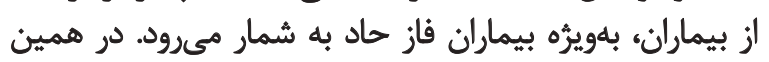

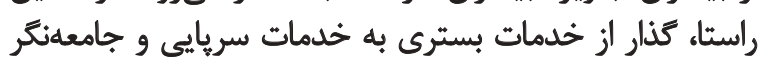
2. Continuity of care

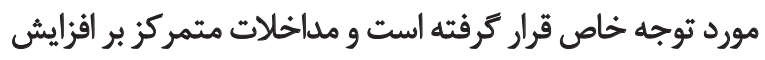

3. Community Mental Health Centers (CMHC)

4. Day Centers

5. Discharge Planning Services

1. Burden of Diseases

\section{.}

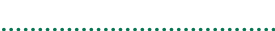

: نويسئده مسينول:

دكتر بهرنك شادلو

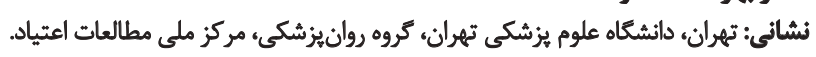

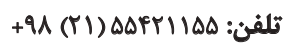
behrang.shadloo@gmail.com : تلفن الكترونيكي 
منابع با استفاده از شيوههاى آمار توصيفى به صورت نمودارهايى

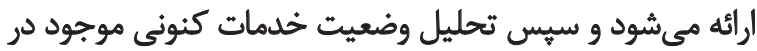

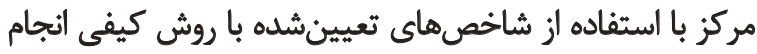

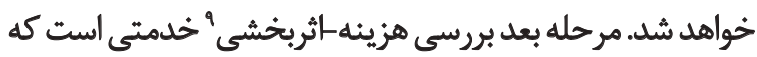

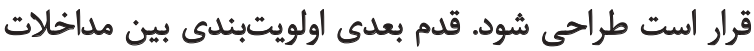

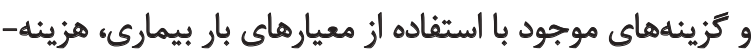

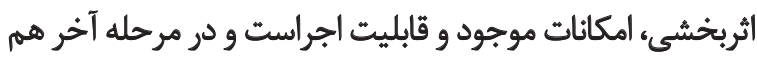
ساختار و جزئيات خدمت مدنظر طراحي مى مابليت اجرود.

كفتنى است دو مرحله اخير (اولويتبندى و طراحى مداخلات)

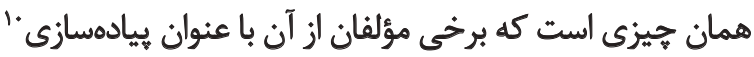

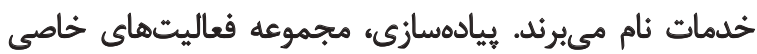

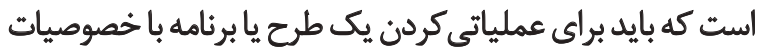

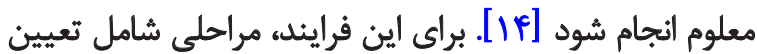

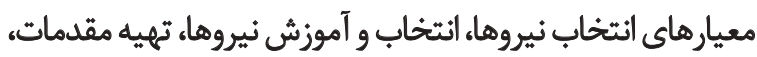

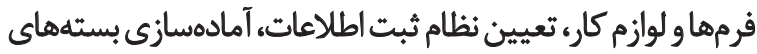

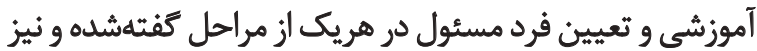

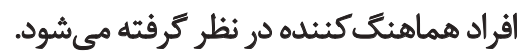

يافتهها

يافتهاى اين يُؤهش در قالب بخشهاى ينج إنهاى كه ذكر

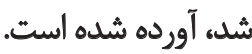

نياززسنى

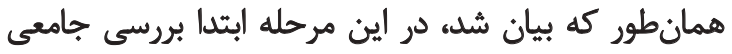

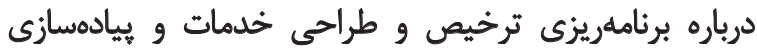

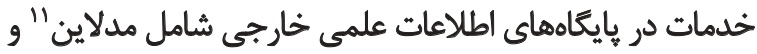

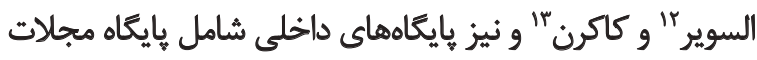

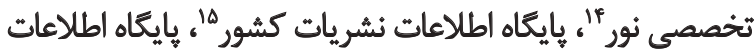

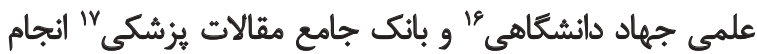

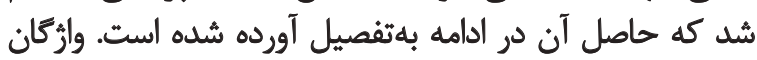

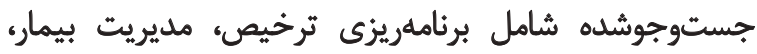

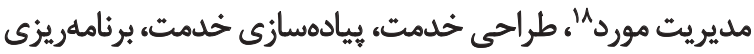

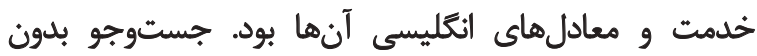
محدوديت زمانى و بدون محدوديت در نوع مطالعه انجام شد. بدا.

\section{Cost-Effectiveness}

10. Implementation

11. Medline

12. Elsevier

13. Cochrane

14. http://www.noormags.ir/

15. http://www.magiran.com/

16. http://sid.ir/

17. http://www.medlib.ir

18. Case management
است نيازمند ارائه و يويكيرى مداخلاتى از قبيل مصرف داروها،

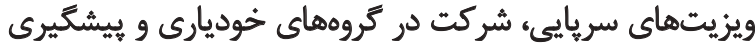

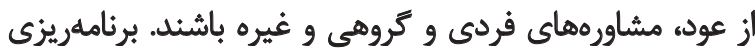

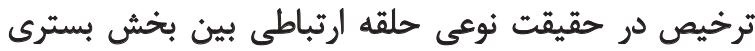

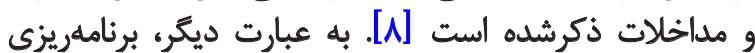

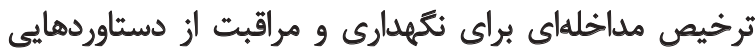

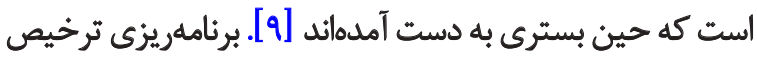

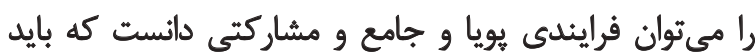

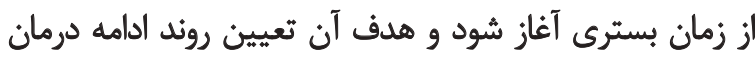

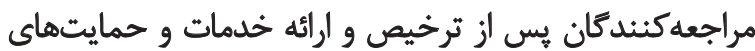

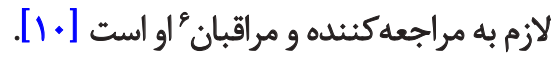

بر اساس مطالعات مرورى جامع و متعددى كه در ادامه آورده

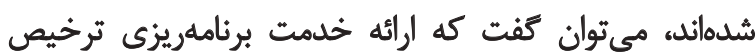

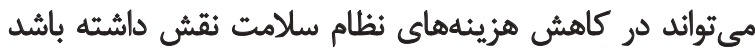

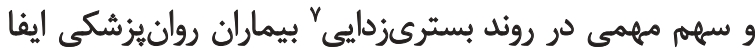

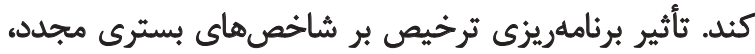

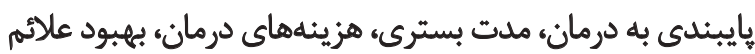

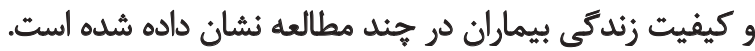

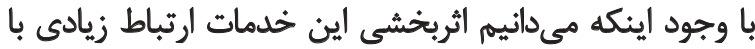

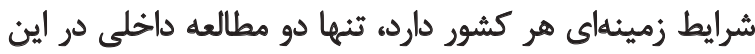

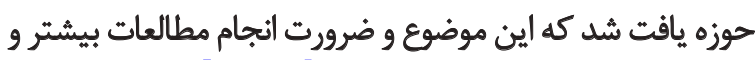

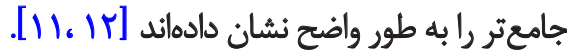

بيمارستان روزبه، وابسته به دانشكاه علوم بزشكى تهران

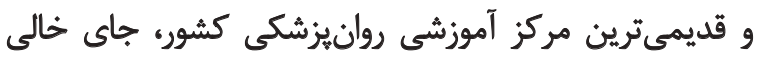

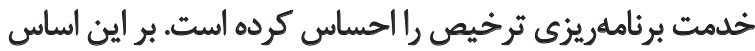

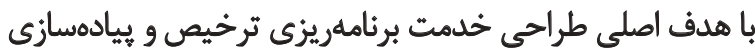

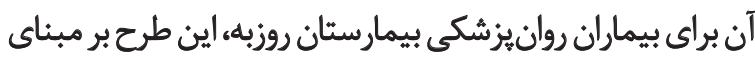

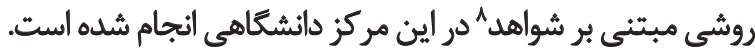

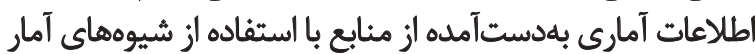

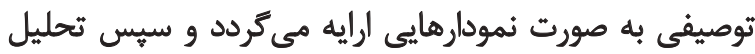

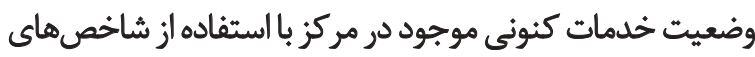
تعيينشده با روش كيفى انجام خواهد شدوند مرند

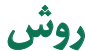

براى طراحى خدمت برنامهريزى ترخيص از الكوى طراحي

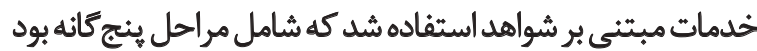

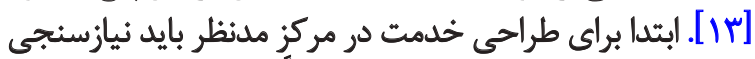

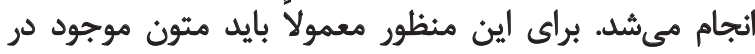

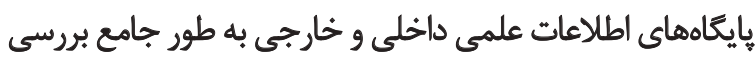

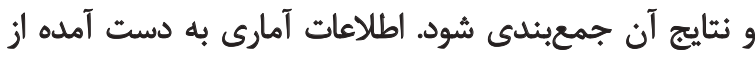

7. Deinstitutionalization

8. Evidence Based 
و همكاران در مطالعه موردى شاهدى تأثير برنامهريزى درمان

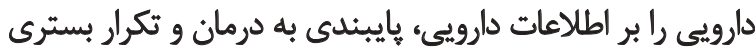

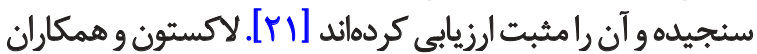

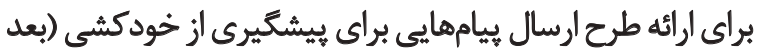

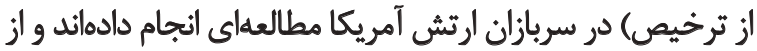

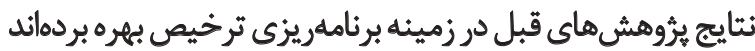

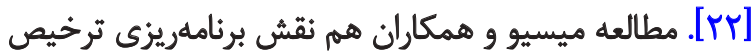

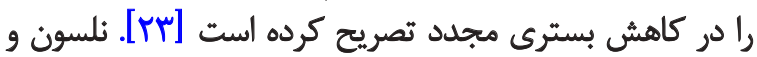

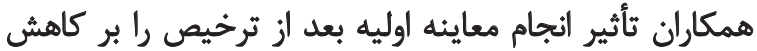

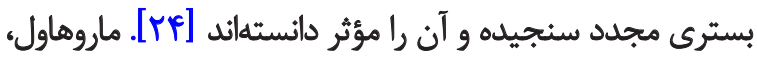

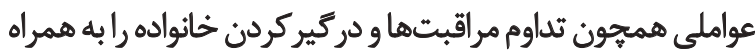

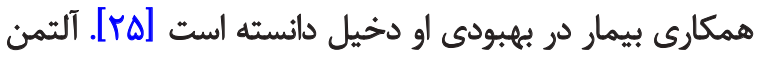

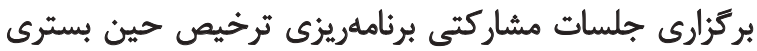

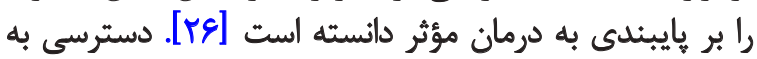

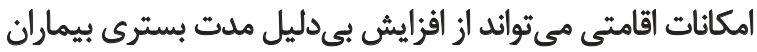

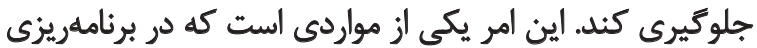

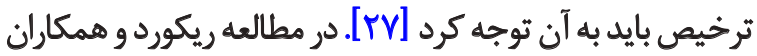

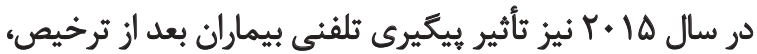

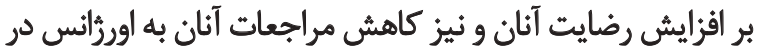

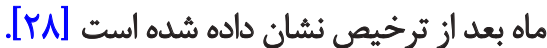

در ايران نيز دو مطالعه در اين زمينه يافت شد. يُروهش رنجبر

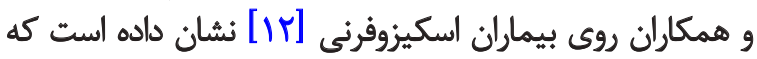

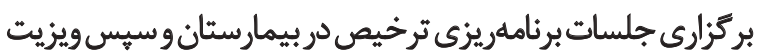

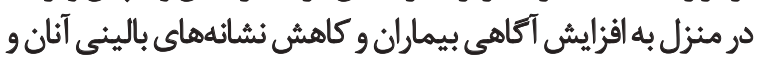

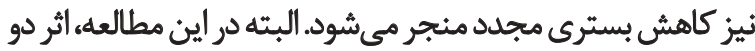

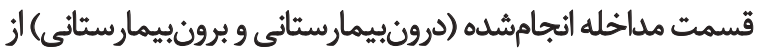

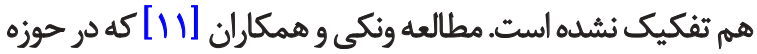

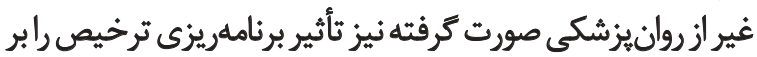
افزرايش رضايت بيماران تأييد كرده است

باوجوداين حقيقت كهاثربخشى اين كونه خدمات، ارتباط زيادى

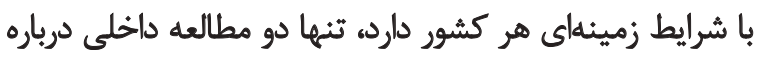

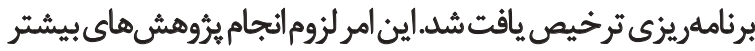

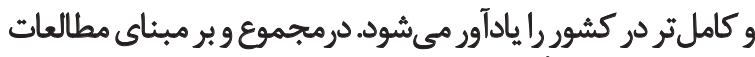

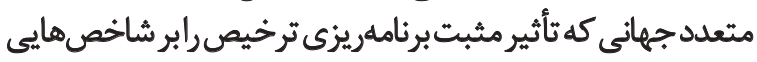

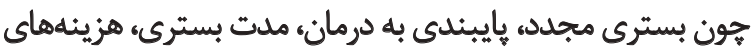

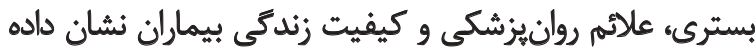

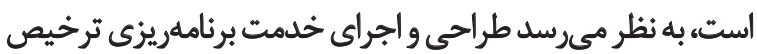

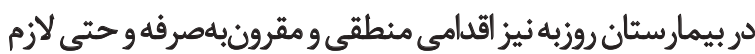

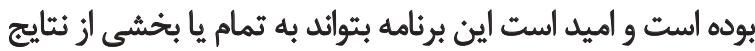
مثبت ذكرشده در مطالعات اشارهشده دست بئ بيدا كند.

تحليل وضعيث موجود

براى توصيف وضعيت كنونى بيمارستان روزبه از نظر نياز به
با توجه به تعداد كم مقالات، نيازى نشد از ميان مقالات انتخاب

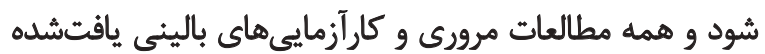
بروسى شنئ.

بر اساس مطالعه مرورى استفن و همكاران كه 11 مطالعه برديه

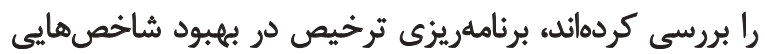

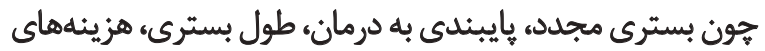

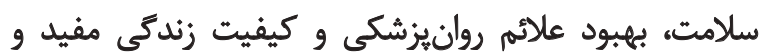

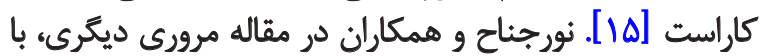

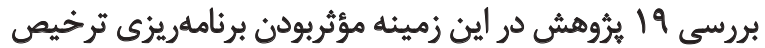

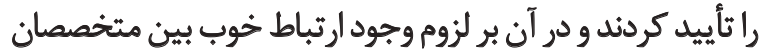

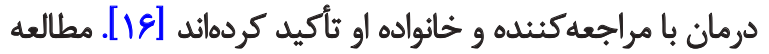

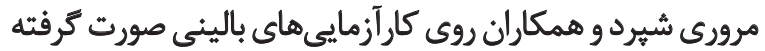

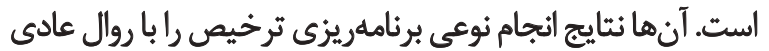

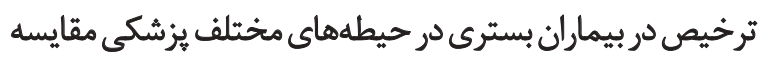

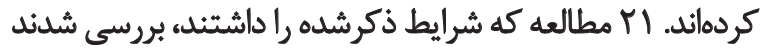

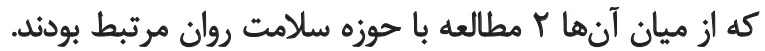

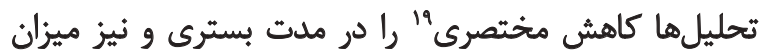

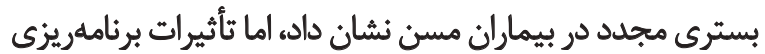

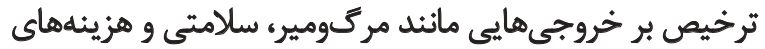

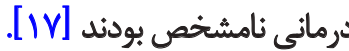

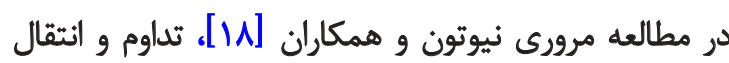

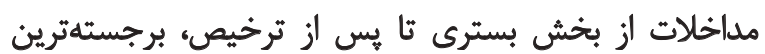

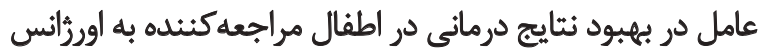

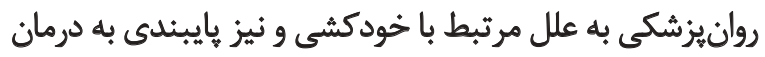
آنان شناخته شده است. مطالعه مرورى فونتانياناروزا و همكارئن

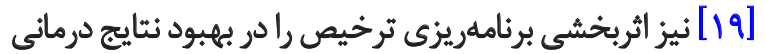

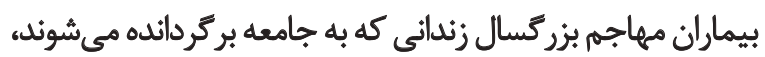

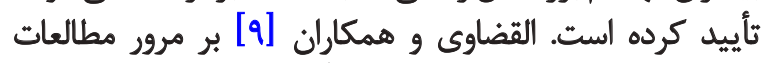

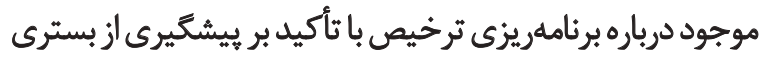

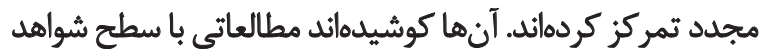

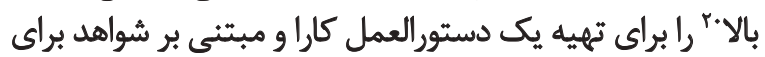

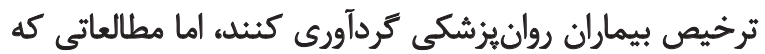

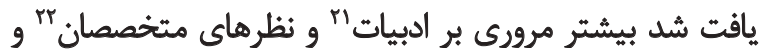
مطالعات توصيفى بآبودئد.

مطالعات ديكرى نيز در ادبيات علمى وجود داشتند كه هريك

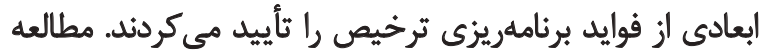

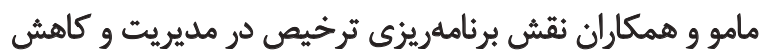

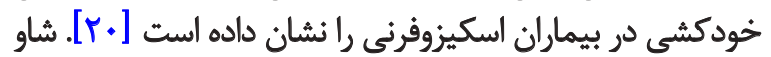

19. A small reduction

20. High level of evidence

21. Literature review

22. Consensus statement

23. Descriptive studies 


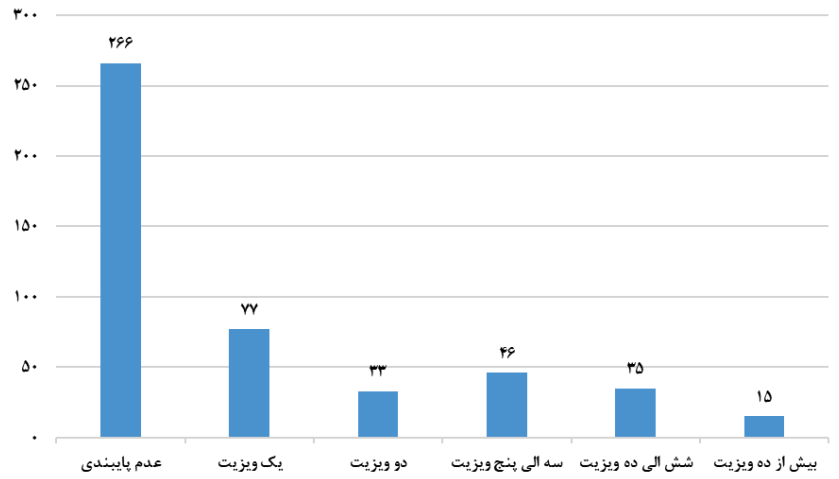

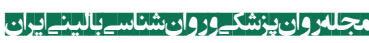

تصوير ا. تعداد مراجعات سريايع بيماران ترخيصشده از بيمارستان روزبه در فاصله ارديبهشت ثا تير كوجا در ششماهه اول ثي از ترخيص

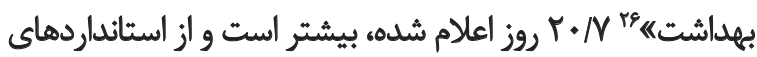

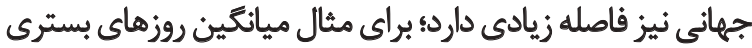

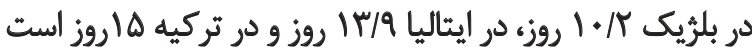

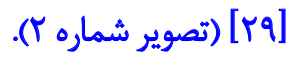

$$
\text { تعيين هزئه -اثربْشُشى خدماث }
$$

باتوجه بل نبود مطالعات مرتبط داخلى، تعيين هزينه-ثربخشى ئري

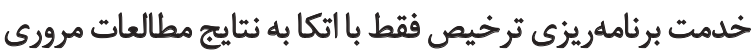

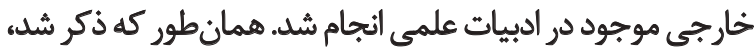

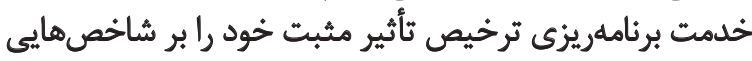

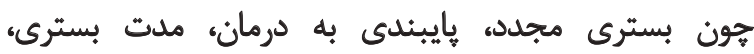

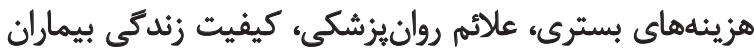

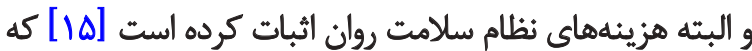

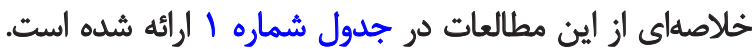

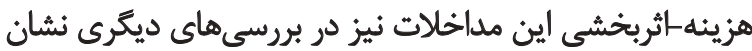

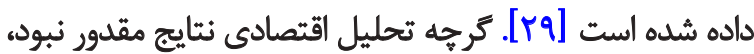

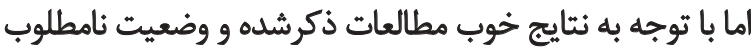

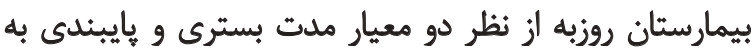

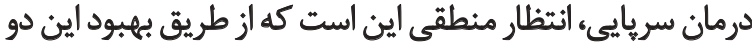

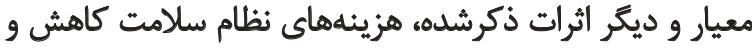
كيفيت خدمات ارتثا يابد.

أنتخاب مداخلات

براى اين كار ابتدا اولويتهاى خدماتى تعيين و سيس ساختار

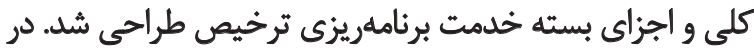

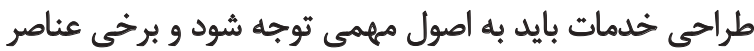

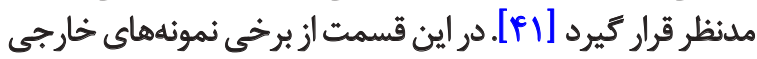

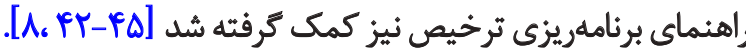

26. World Health Organization Assessment Instrument for Mental Health Systems (WHO-AIMS)

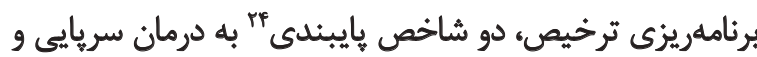

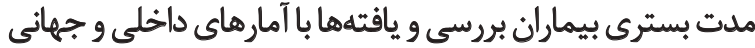
مقايسه شد.

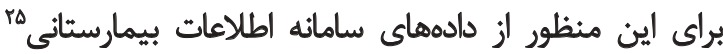

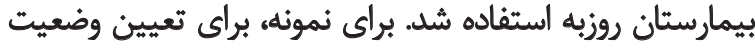

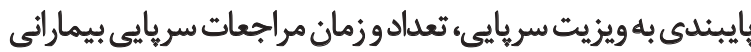

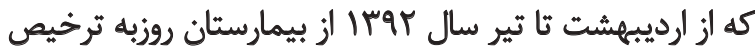

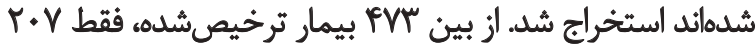

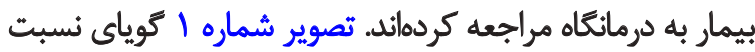

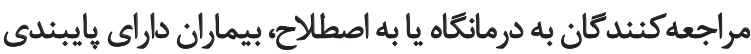

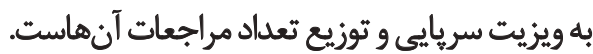

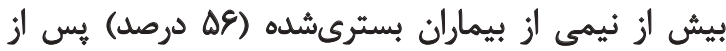

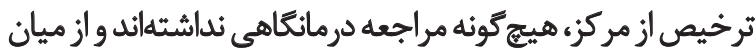

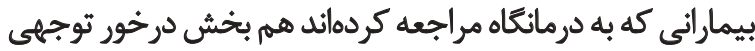

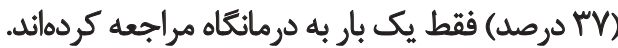

با توجه به اينكه در مطالعات مرورى و بدون انجام برنامهريزى

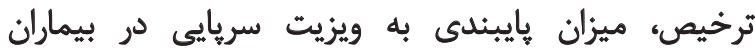

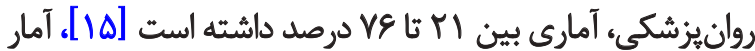

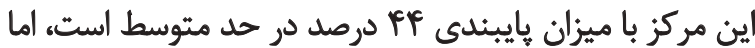

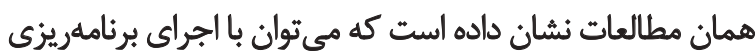

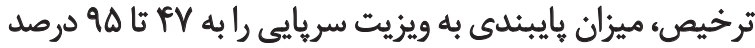

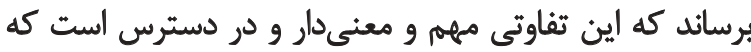
مي تونواند در اهداف برنامه قرار كيرد. همجنين با بررسى دادههاى سامانه اطلاعات بيمارستانى،

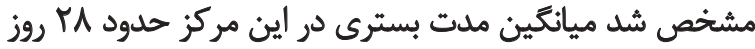

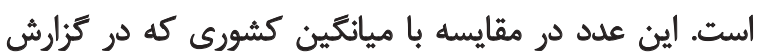

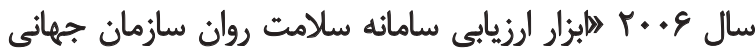

24. Adherence

25. Hospital Information System (HIS) 


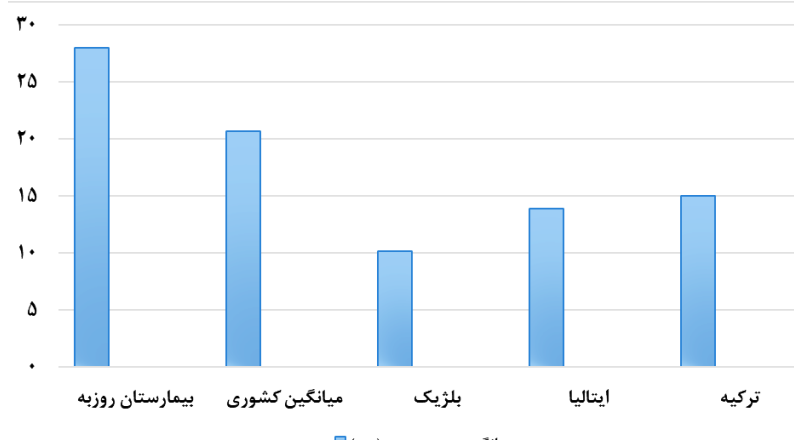

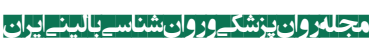

تصوير r. ميائكين مدت بسترى بيماران در بيمارستان روزبه و مقايسه آن با آهار داخلى و جهانى

با توجه به مطالعات ذكرشده، براى طراحى خدمت حمايتى مورد نياز بيمار در جامعه، مداخله زودرس در بحران يا

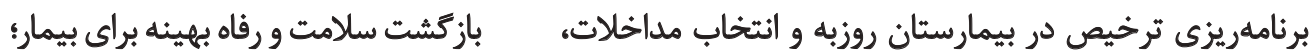

• براي اطمينان از اينكه بيمار بيشترين ميزان رضايتمندى و روفاه به اصول زير دقت شد: برحئ

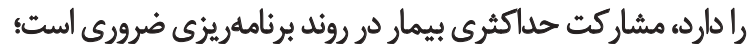

•خانواده يا ديكر مراقبان بيمار نيز بايد تا جاي ممكن در روند

• هدف اصلى از برنامهريزى ترخيص، اطمينان از اين موارد

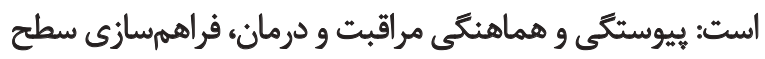

جدول ا. خلاصهاى ازمطالعات انجامشده در حوزه برنامهريزى ترخيص، بركرفته از مطالعه استفن و همكاران [ه ا ]

\begin{tabular}{|c|c|c|c|c|c|}
\hline ملام هاي بروسي شده & طراحي مطالعه & حجميم نموند & مؤلف و سال اجرا & 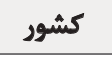 & رديف \\
\hline هيزان استفاده از خلدمات سلامت روان و ملت بسترى & كوهورت & (9) & Cuffel, 2002 [31] & أهريكا & 1 \\
\hline كيفيت زندكى و هزينهاى نظام سلامت & كارآزمايي باليني تصادفي & rq. & $\begin{array}{l}\text { Forchuk, } 2005 \\
\qquad[32]\end{array}$ & كانادا & r \\
\hline يايبندى به درمان و بسترى مجلد & كارآزمايي بالينى & Vo & $\begin{array}{l}\text { Karniel-Lauer, } \\
2000[33]\end{array}$ & أسرائيل & r \\
\hline بسترى مجلد و يايبندى به ويزيت اول يس از ترخيص & كارآز كمايع باليني تصادفى & mer & Naji, 1999 [34] & |نكلستان & f \\
\hline 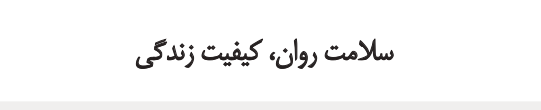 & كارازئي بالينى تصادفى & m & Naoki, 2002 [35] & رائن & $\Delta$ \\
\hline بسترى مجلد & 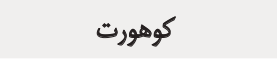 & Arq & Nelson, 2014 [24] & أمريكا & 8 \\
\hline بسترى مجدد، سلامت روانه كيفيت زندكى و بايبيثلدى به & كارآزهايي بالينى كتثرل شده & 11. & Olfson, 1998 [36] & آهريكا & $\checkmark$ \\
\hline هايبندى به ويزيتهاى يس از ثترخيص & 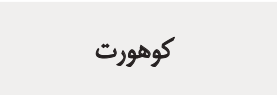 & milv & $\begin{array}{l}\text { Orlosky, } 2007 \\
{[37]}\end{array}$ & 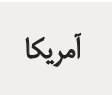 & $\wedge$ \\
\hline بسترى هجدد و سلامت روان و كيفيت زندكى & كار كتثرل بشلينى تصادفى & 19 & $\begin{array}{l}\text { Reynolds, } 2004 \\
\text { [38] }\end{array}$ & 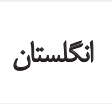 & 9 \\
\hline هايبندى به ويزيت اول يس از ترخيص & كارآزهايي بالينى كنترل شده & Ar & $\begin{array}{l}\text { Sharma, } 1995 \\
\text { [39] }\end{array}$ & آهريكا & 1. \\
\hline بسترى مجلد و مشكلات دارويى & كار آزمايى بالينى تصادفى & 9v & Shaw, 2000 [21] & ان انغلستان & 11 \\
\hline ياييثلى به ويزيت اول يس از ترخيص & مطالعه تصادفى شلده" & $|r|$ & $\begin{array}{c}\text { Swanson, } 1999 \\
{[40]}\end{array}$ & 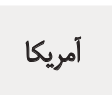 & ir \\
\hline
\end{tabular}

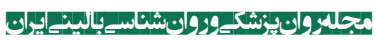

1. Randomized Controlled Trial (RCT); 2. Controlled Clinical Trial (CCT); 3. Randomized Trial (RT) 


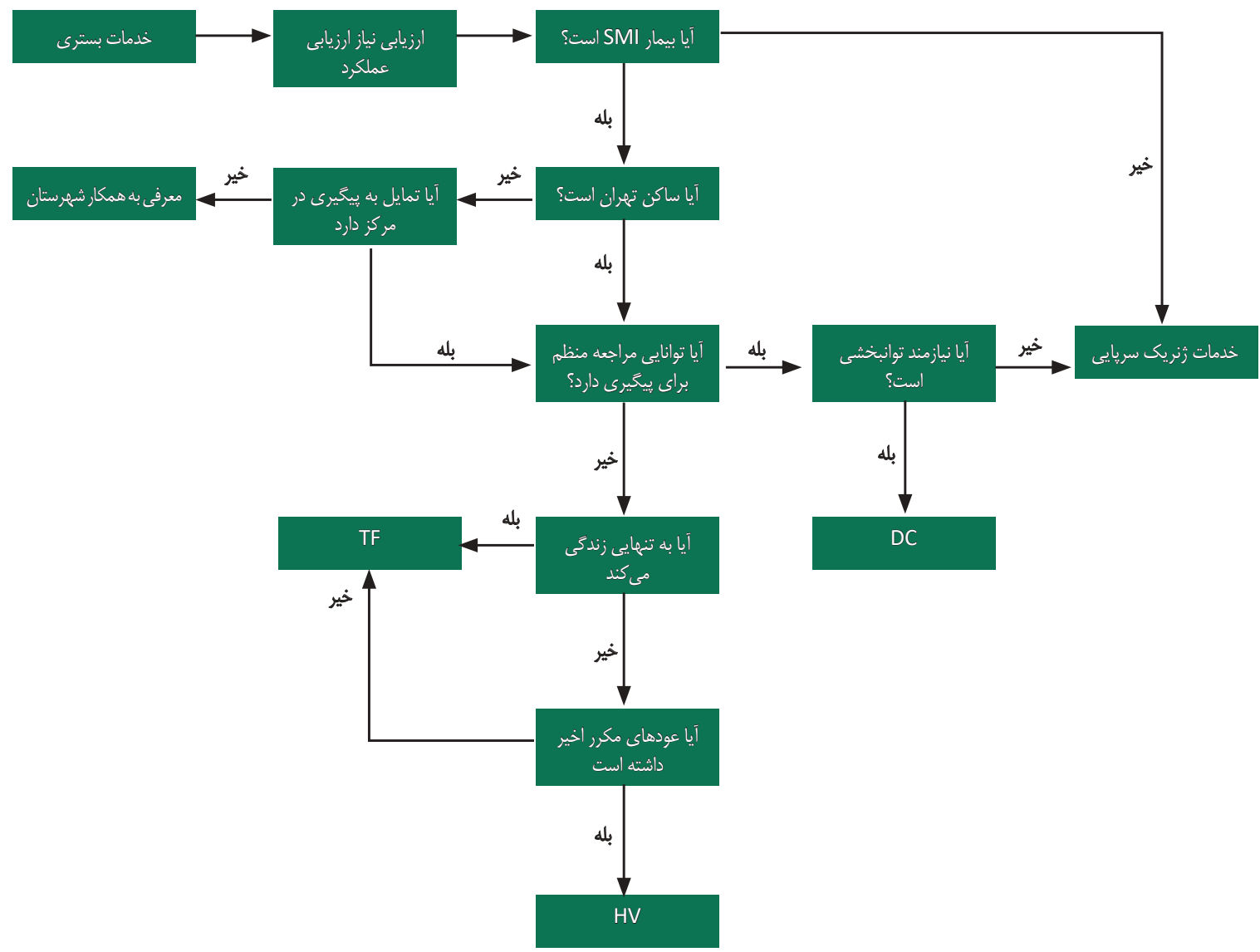

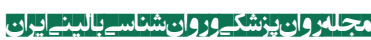

تصوير ז. فرايثل برنامهريزى ترخيص در بيمارستان روزبه

$$
\text { اقامتي موردنياز را يايش كنئد. }
$$

درمان مشاركت دائتئه باشُشند؛

عناصر مهمي كه در طراحي اين خدمات مدنظر قرار كرفت

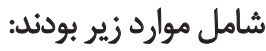

• توجه به ماهيت مزمن و عودكنيله و ناتوانكنيده غالب

$$
\text { بيمارىهاى روانيزشكى؛ ماهئ }
$$

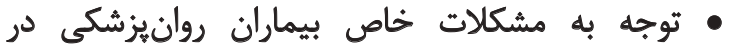

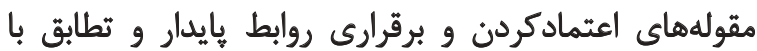
شرايط نامساعد يا جديد؛

• داشتن جامعيت كافي در شُ حوزه مهرهم مسائل بيماران

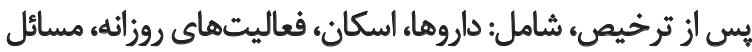
طبى، آموزش و ديكر حمايتها و نحوه ارتباط با تيهم درمان؛ • مشاركتي بودن خدمات، شامل ارائه خدمات به صورت

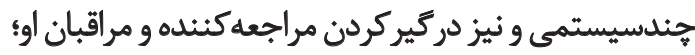
• تداوم خدمات در سه حوزه اصلي اطلاعاتي و مديريتي

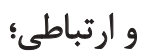
• مستندسازى تمامى اطلاعات جمعآورىشده و ارائهده و دستور العمل ها و برنامههاي آينده؛
• مراقبتهايى كه در خلال برنامهريزى ترخيص مطرح

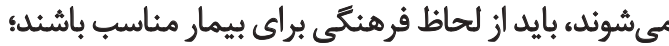
• ارزيابى نيازهاى بيمار، ازجمله اقدامات مقدماتي در برناملهيزى ترخيص است؛

• مسائل بالينى و طبى مرتبط بايد در روند برنامهريزى ترخيص بإني ملنظر باشند؛

• برنامهريزى ثرخيص بايل به صورت فردى براي هر بيمار

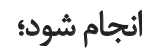

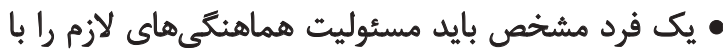

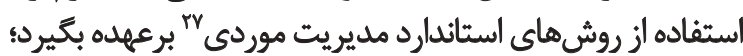
• در سطح مديريت، فراهم كنيندكان خدمات سلامت روان بايد

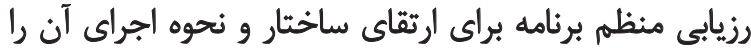

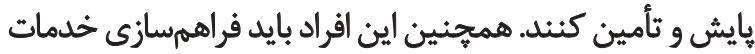

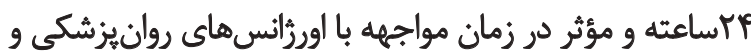

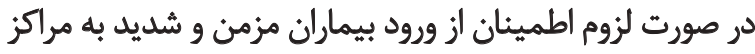

27. Case management 


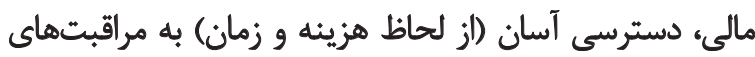

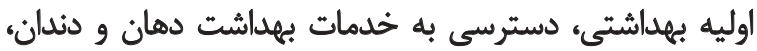

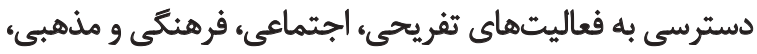

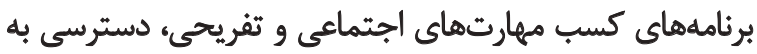

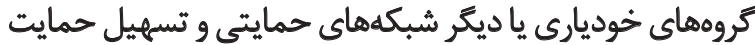

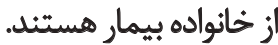

r. هماهنكى خدمات: براى هريك از نيازهاى تعيينشانده، بايد

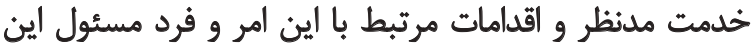

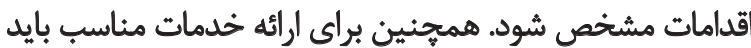

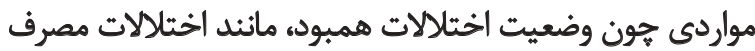

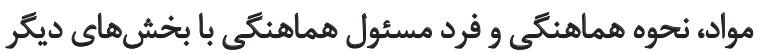

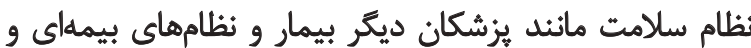

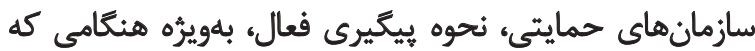

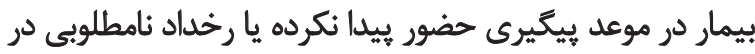

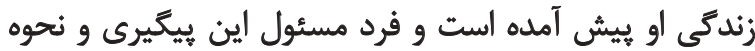

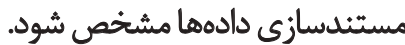
f. فهرستى از اقدامات نظارتى و مسائل مرتبط با بيمار: داشتن

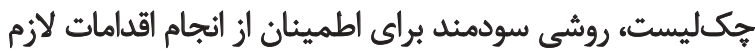

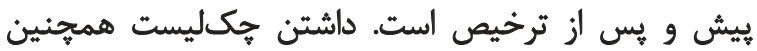

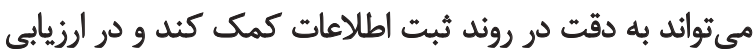
خدمات هم استفاده شُود. طرامي خمدمت

در طراحى هر خدمت جديد، بايد اجزاي آن بهدقت مشخص و

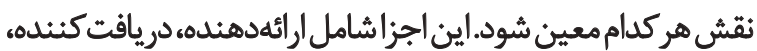

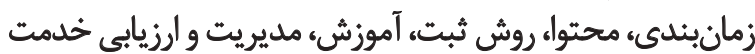
مور نيظر هيستند.

اجزاى خدمات برنامهريزى ثرخيص در بيمارستان روزبه

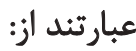
ارائهدهئه خدمث

با وجود اينكه فرايند برنامهريزى ترخيص دربركيرنده افراد

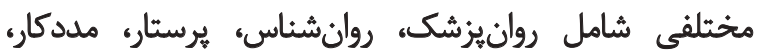

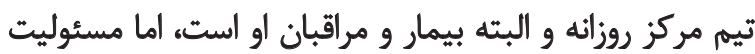
هماهنكي كلى برعهلده يك نفر (رابط درمان) است.

\section{دريافت كنيده خدمت}

اغلب خود بيمار و در مراحل بعدى مراقبان و اطرافيانش، تروه هدف خدمات برنامهريزى ترخيص هستيمارود مراحل بعدي مربان مدل و زمانبئدي خحمث فرايند كلى برنامهريزى ترخيص در بيمارستان روزبه در تصوير
• •تعيين مسئول مستقيم براي بيكيرى امور و ياسخ كويى به هر مراجعه كنيده بعد از ترخيص.

درنهايت يُ از جمعبندى و مرور مداخلات و ابزارهاي موجود

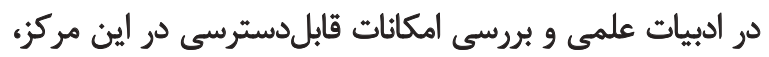
مانيد بودجه، و لوازم و ابزار و ونيروى انسانى موريات موردئياز، تصميم

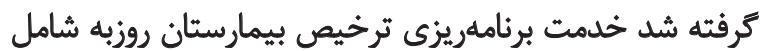
جهار قسمت زير باشد:

1. ثبت مشخصات بيمار: كرفتن و ثبت اطلاعات بيمار در

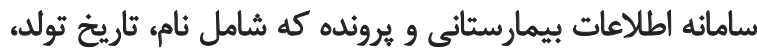

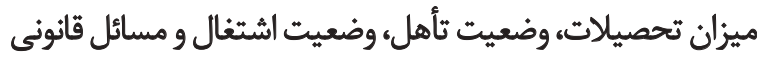

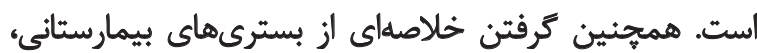

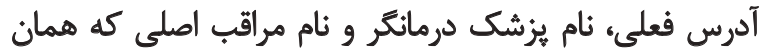

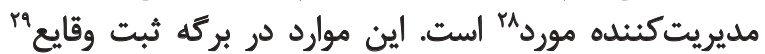

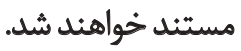
r. ابزارهايى براى ارزيابى نيازها: ابزارهاى موردنظر بايد سيه

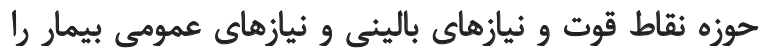

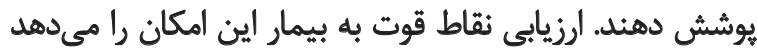

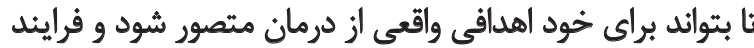
توانبخشى را با رضايت و موفقيت بيشترى سيرى كندي اندي

درباره نيازهاى بالينى، نظر به اينكه هر بيمار بسته به ماهيت

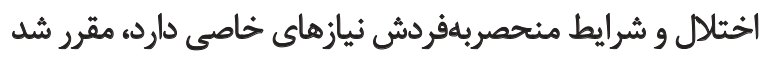

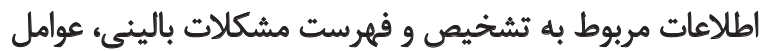

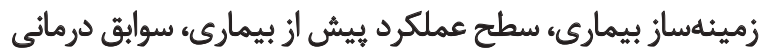

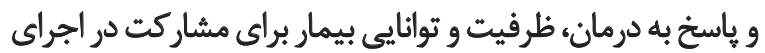

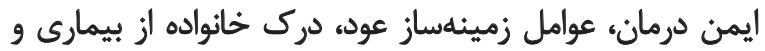

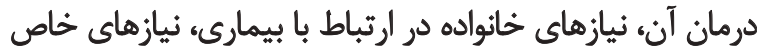

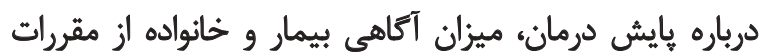

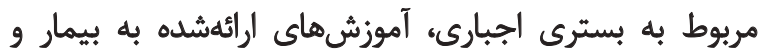

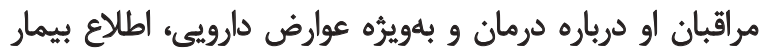

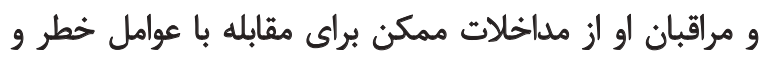

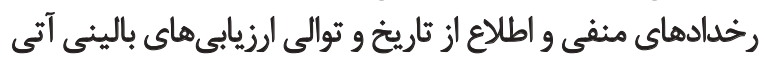

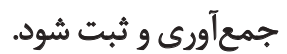

نيازهاى عمومى بيماران كه هنكام تدوين برنامه ترخيص بايد

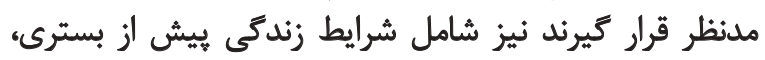

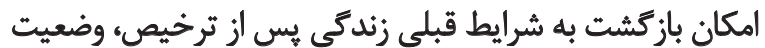

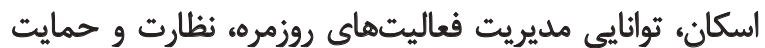

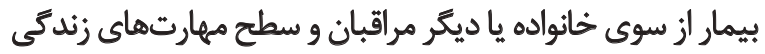

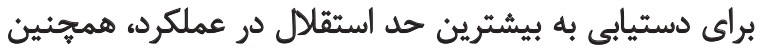

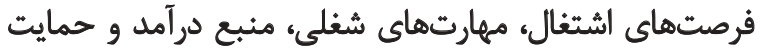

28. Case manager

29. Incident report 


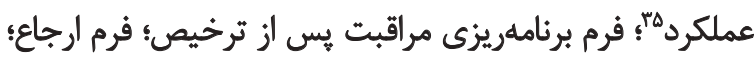

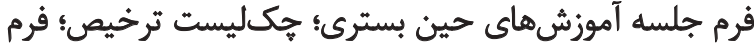

برئامهريزى ترخيص بيمار.

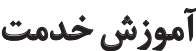

طبق آنجه مقرر شده است قبل از اجراى آزمايشى خدمات در

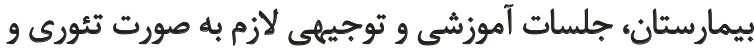

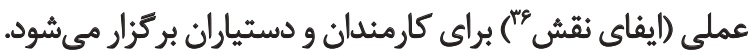

\section{مديريت خدمت}

مسئول آموزش بيمار فرمهاى برنامهريزى ترخيص رابه صورت

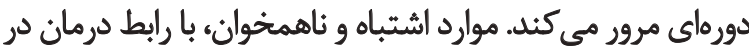

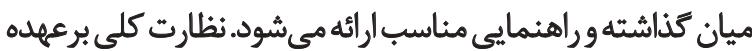

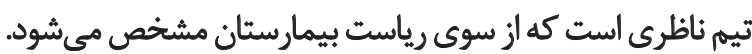

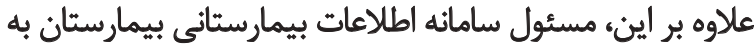

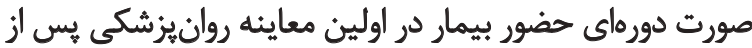

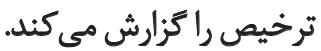

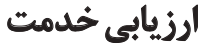

شاخصهاى اصلى ارزيابى براى برنامهريزى ترخيص شامل

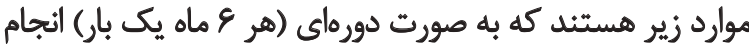

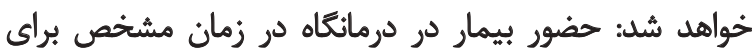

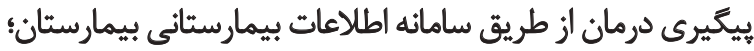

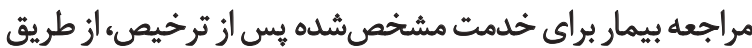

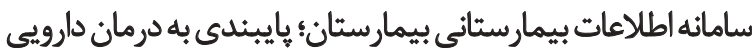

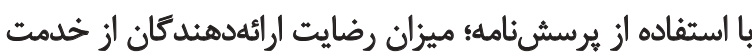

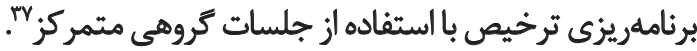

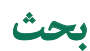

برنامهريزى ترخيص بلهمثابه يك مداخله بالينى، جزئي از درمان

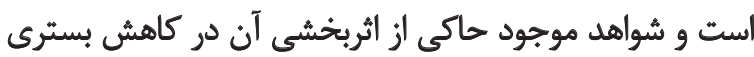

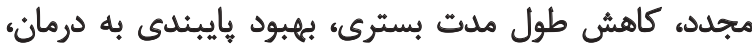

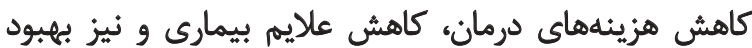

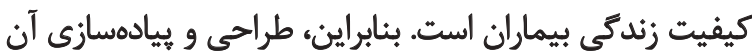

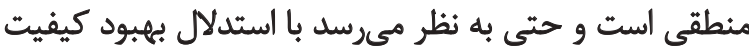
خدمات سلامت، يك الزام اخلاقى باشد. طراحى خدمات برنامهريزى ترخيص در بيمارستان روزبه بر بر باري

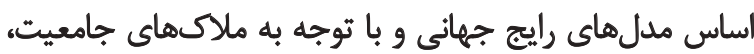

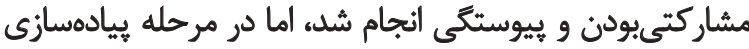

35. Global Assessment of Functioning (GAF)

36. Role play

37. Focused group sessions

$$
\text { شماره ب ترسيم شده است. }
$$

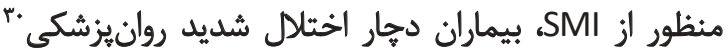

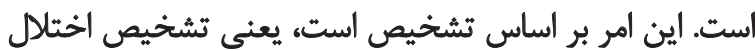

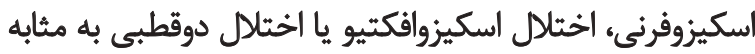

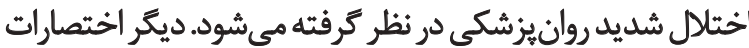

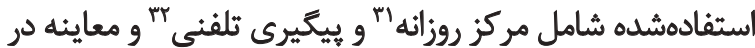

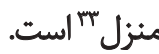

محتواى خدمت

خدمت برنامهريزى ترخيص حين بسترى براى هر بيمار در

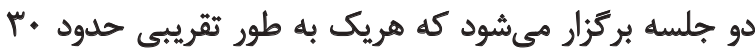

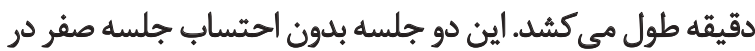

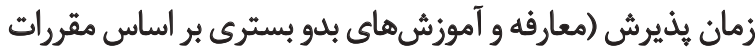

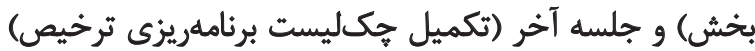

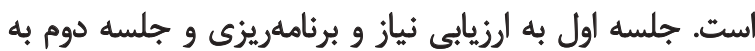

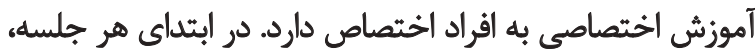

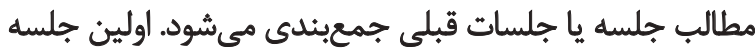

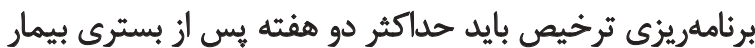

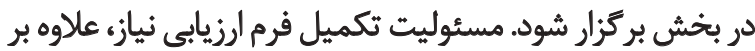

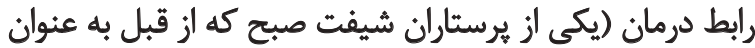

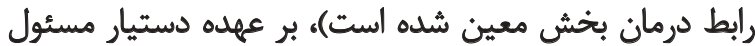

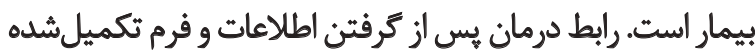

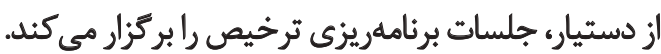

تعيين شهر محل اسكان بيمار و تمايل بيمار براى بييخيرى

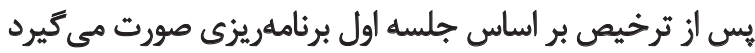

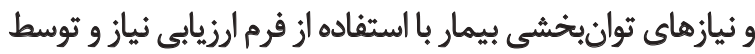

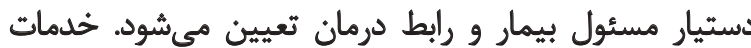

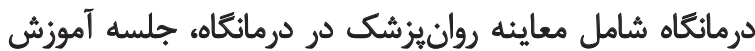

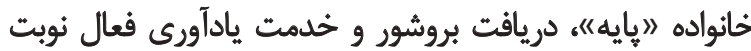

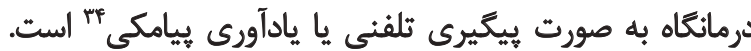

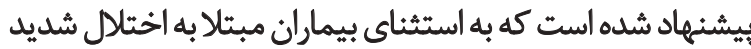

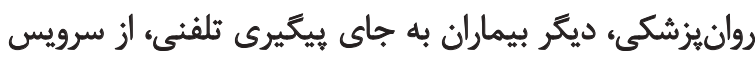
و بيامكى يادآورى نوبت استفاده كنئد.

$$
\text { روش ثبت خدمت }
$$

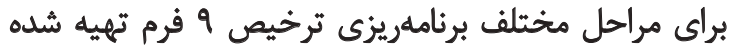

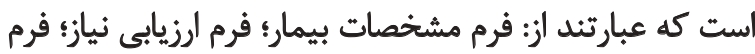

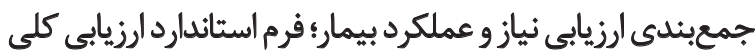

30. Severe mental illness

31. Day Center (DC)

32. Telephone Follow up (TF)

33. Home Visit (HV)

34. SMS reminder 
لازم مى دانند از كمكهاى همكاران محترم، خانم دكتر زهرا

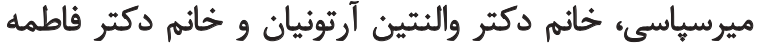

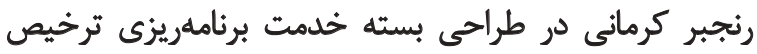

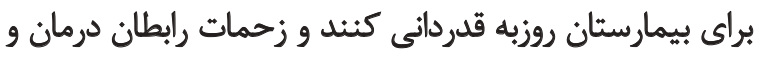

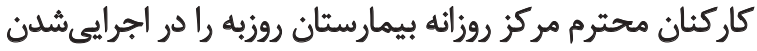

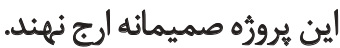

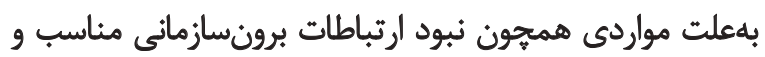

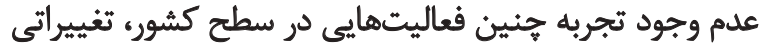

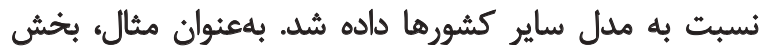

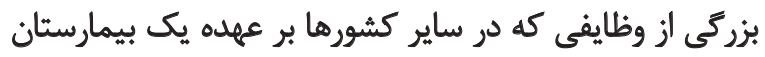

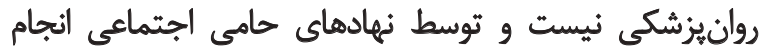

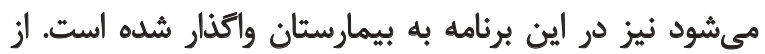

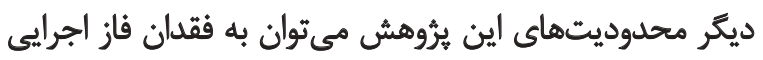

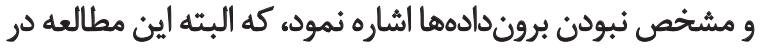
حال انجام است ودر آينده نتايج آن منتشر خواهد شد. ندود

\section{نتيجهئيرى}

برنامهريزى ترخيص، نوعى مداخله بالينى و جزئي از درمان

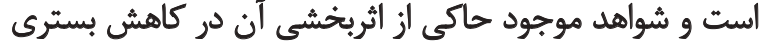

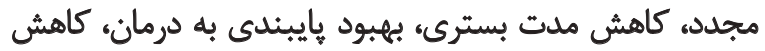

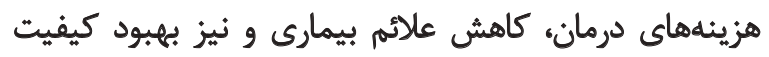

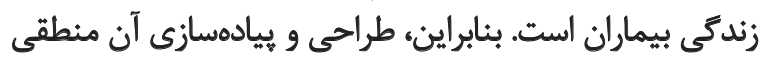

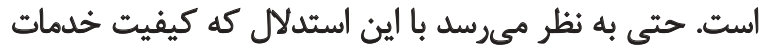
سلامت بايد بهبود يابد، يك الزام اخلاقى باشد.

طراحى خدمات برنامهريزى ترخيص در بيمارستان روزبه بر بران

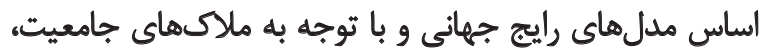

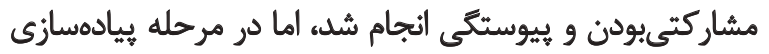

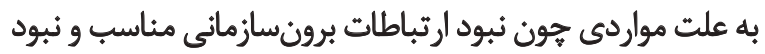

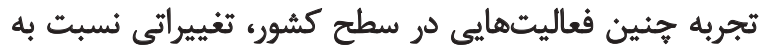

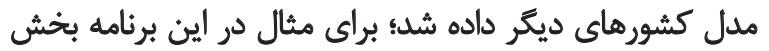

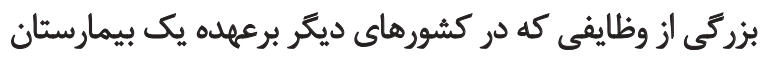

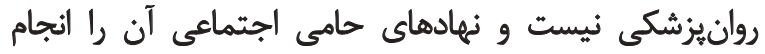

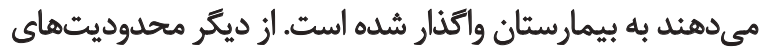

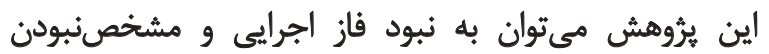

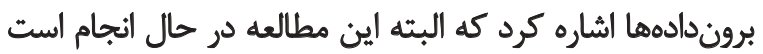

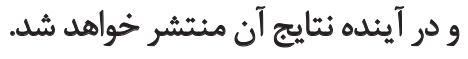

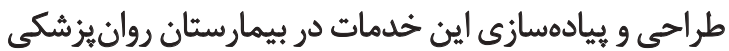

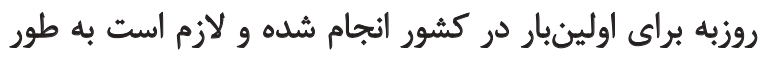

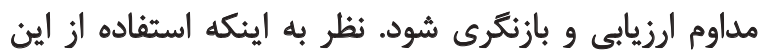

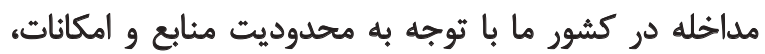

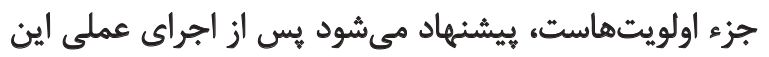

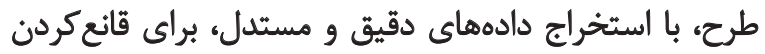

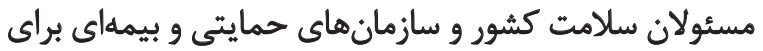

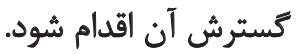

$$
\text { سياسكّزارى }
$$

اين مطالعه برترفته از هايانثامه دوره دستيارى تخصصى

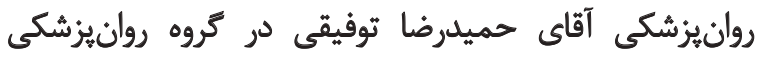

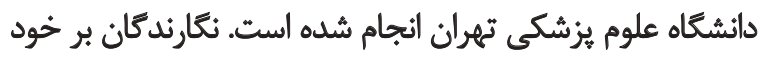




\section{References}

[1] Vandad Sharifi M, Hajebi A, Radgoodarzi R. [Twelve-month prevalence and correlates of psychiatric disorders in Iran: The Iranian mental health survey, 2011 (Persian)]. Archives of Iranian Medicine. 2015; 18(2):76.

[2] Kessler RC, Berglund P, Demler O, Jin R, Merikangas KR, Walters EE. Lifetime prevalence and age-of-onset distributions of DSM-IV disorders in the national comorbidity survey replication. Archives of General Psychiatry. 2005; 62(6):593. doi: 10.1001/ archpsyc.62.6.593

[3] Mathers C, Boerma T, Fat DM. The global burden of disease. Geneva: World health organisation; 2008.

[4] Bloom DE, Cafiero E, Jané Llopis E, Abrahams Gessel S, Bloom LR, Fathima S, et al. The global economic burden of noncommunicable diseases. Harvard: Program on the Global Demography of Aging; 2012.

[5] Lamb HR, Bachrach LL. Some perspectives on deinstitutionalization. Psychiatric Services. 2001; 52(8):1039-45. doi: 10.1176/ appi.ps.52.8.1039

[6] Van Walraven C, Oake N, Jennings A, Forster AJ. The association between continuity of care and outcomes: a systematic and critical review. Journal of Evaluation in Clinical Practice. 2010; 16(5):947-56. doi: 10.1111/j.1365-2753.2009.01235.x

[7] Sharifi V, Abolhasani F, Farhoudian A, Amin Esmaeili M. [Community mental health centers in Iran: Planning evidence-based services (Persian)]. Iranian Journal of Psychiatry \& Clinical Psychology. 2013; 19(3):163-176

[8] Baron M, Erlenbusch B, Moran CF, O'Connor K, Rice K, Rodriguez J. Best practices manual for discharge planning: Mental health \& substance abuse facilities, hospitals, foster care, prison and jails. Los Angeles: Coalition to hunger \& homelessness. 2008.

[9] Alghzawi HM. Psychiatric discharge process. ISRN Psychiatry. 2012; 2012:1-7. doi: 10.5402/2012/638943

[10] Kerr MS. Psychiatric discharge summaries. BMJ. 1990; 300(6719):260-1. doi: 10.1136/bmj.300.6719.260-b

[11] Vanaki Z, Habibipour B. [The assessment of effect discharge planning on patients satisfaction (Persian)]. Scientific Journal of Hamadan Nursing \& Midwifery Faculty. 2008; 16(2):25-35.

[12] Ranjbar F, Ghanbari B, Khaleghparast S, Manouchehri H, Nasiri $\mathrm{N}$. [The effects of discharge planning on insight, symptoms and hospitalization of Schizophrenia patients at Iran University of Medical Sciences Hospitals (Persian)]. Iranian Journal of Psychiatry and Clinical Psychology. 2011; 17(1):53-9.

[13] Edvardsson B, Olsson J. Key concepts for new service development. The Service Industries Journal. 1996; 16(2):140-64. doi: $10.1080 / 02642069600000019$

[14] Fixsen DL, Naoom SF, Blase KA, Friedman RM. Implementation research: A synthesis of the literature. Tampa, FL: University of South Florida; 2005.

[15] Steffen S, Kösters M, Becker T, Puschner B. Discharge planning in mental health care: A systematic review of the recent literature. Acta Psychiatrica Scandinavica. 2009; 120(1):1-9. doi: 10.1111/j.1600-0447.2009.01373.x

[16] Nurjannah I, Mills J, Usher K, Park T. Discharge planning in mental health care: An integrative review of the literature. Jour- nal of Clinical Nursing. 2013; 23(9-10):1175-85. doi: 10.1111/ jocn. 12297

[17] Shepperd S, McClaran J, Phillips CO, Lannin NA, Clemson LM, McCluskey A, et al. Discharge planning from hospital to home.In: Shepperd S, editor. Cochrane database of systematic reviews. Hoboken, NJ: John Wiley \& Sons, Ltd; 2010. doi: 10.1002/14651858.cd000313.pub3

[18] Newton AS, Hamm MP, Bethell J, Rhodes AE, Bryan CJ, Tjosvold $\mathrm{L}$, et al. Pediatric suicide-related presentations: A systematic review of mental health care in the emergency department Annals of Emergency Medicine. 2010; 56(6):649-659.e2. doi: 10.1016/j.annemergmed.2010.02.026

[19] Fontanarosa J, Uhl S, Oyesanmi O. Interventions for adult offenders with serious mental illness [Internet]. Rockville (MD): Agency for Healthcare Research and Quality (US); 2013 Aug. Report No.: 13-EHC107-EF.

[20] Mamo DC. Managing suicidality in schizophrenia. Canadian Journal of Psychiatry. 2007; 52(6):59S. PMID:17824353

[21] Shaw H, Mackie CA, Sharkie I. Evaluation of effect of pharmacy discharge planning on medication problems experienced by discharged acute admission mental health patients. International Journal of Pharmacy Practice. 2000; 8(2):144-53. doi: 10.1111/ j.2042-7174.2000.tb00999.x

[22] Luxton DD, Thomas EK, Chipps J, Relova RM, Brown D, McLay R, et al. Caring letters for suicide prevention: Implementation of a multi-site randomized clinical trial in the U.S. military and veteran affairs healthcare systems. Contemporary Clinical Trials. 2014; 37(2):252-60. doi: 10.1016/j.cct.2014.01.007

[23] Missio H. Does discharge planning prevent readmission to inpatient psychiatric units. Gosford, New South Wales: Central Coast Health; 2004

[24] Nelson EA, Maruish ME, Axler JL. Effects of discharge planning and compliance with outpatient appointments on readmission rates. Psychiatric Services. 2000; 51(7):885-9. doi: 10.1176/ appi.ps.51.7.885

[25] Morrow Howell N, Proctor EK, Mui AC. Adequacy of discharge plans for elderly patients. Oxford: Oxford University Press; 1991.

[26] Altman H. A Collaborative approach to discharge planning for chronic mental patients. Psychiatric Services. 1983; 34(7):641-2. doi: $10.1176 /$ ps.34.7.641

[27] Kelly A, Watson D, Raboud J, Bilsker D. Factors in delays in discharge from acute-care psychiatry. The Canadian Journal of Psychiatry. 1998; 43(5):496-501. doi: 10.1177/070674379804300508

[28] Record JD, Niranjan Azadi A, Christmas C, Hanyok LA, Rand CS, Hellmann DB, et al. Telephone calls to patients after discharge from the hospital: An important part of transitions of care. Medical Education Online. 2015; 20(1):26701. doi: 10.3402/meo v20.26701

[29] Puschner B, Steffen S, Gaebel W, Freyberger H, Klein HE Steinert $\mathrm{T}$, et al. Needs-oriented discharge planning and monitoring for high utilisers of psychiatric services (NODPAM): Design and methods. BMC Health Services Research. 2008; 8(1). doi: 10.1186/1472-6963-8-152

[30] Puschner B, Steffen S, Gaebel W, Freyberger H, Klein HE Steinert $\mathrm{T}$, et al. Needs-oriented discharge planning and monitoring for high utilisers of psychiatric services (NODPAM): Design 
and methods. BMC Health Services Research. 2008; 8:152. doi: 10.1186/1472-6963-8-152

[31] Hansen DC, Matt Hensrud N, Holland DE, Severson MA. Development of a discharge planning mentorship program. Journal for Nurses in Staff Development. 2000; 16(1):11-6. doi: 10.1097/00124645-200001000-00002

[32] Forchuk C, Martin M, Chan YL, Jensen E. Therapeutic relationships: from psychiatric hospital to community. Journal of Psychiatric and Mental Health Nursing. 2005; 12(5):556-64. doi: 10.1111/j.1365-2850.2005.00873.x

[33] ]Naji SA, Howie FL, Cameron IM, Walker SA, Andrew J, Eagles JM. Discharging psychiatric in-patients back to primary care: a pragmatic randomized controlled trial of a novel discharge protocol. Primary Care Psychiatry. 1999; 5(3):109-15.

[34] Naoki K, Nobuo A, Emi I. [Randomized controlled trial on effectiveness of the community re-entry program to inpatients with schizophrenia spectrum disorder, centering around acquisition of illness self-management knowledge (Japanese)]. Seishin Shinkeigaku Zasshi. 2003; 105(12):1514-31. PMID: 15027310

[35] Grant JE. Caring for your patient after discharge. Current Psychiatry. 2007; 6(4):75-7.

[36] Olfson M, Mechanic D, Boyer CA, Hansell S. Linking Inpatients With Schizophrenia to Outpatient Care. Psychiatric Services. 1998; 49(7):911-7. doi: 10.1176/ps.49.7.911

[37] Orlosky MJ, Caiati D, Hadad J, Arnold G, Camarro J. Improvement of Psychiatric Ambulatory Follow-up Care by Use of Care Coordinators. American Journal of Medical Quality. 2007; 22(2):95-7. doi: 10.1177/1062860606297997

[38] Reynolds W, Lauder W, Sharkey S, Maciver S, Veitch T, Cameron D. The effects of a transitional discharge model for psychiatric patients. Journal of Psychiatric and Mental Health Nursing. 2004; 11(1):82-8. doi: 10.1111/j.1365-2850.2004.00692.x

[39] Sharma SB, Elkins D, van Sickle A, Roberts CS. Effect of Predischarge Interventions on Aftercare Attendance: Process and Outcome. Health \& Social Work. 1995; 20(1):15-20. doi: 10.1093/ hsw/20.1.15

[40] Swanson AJ, Pantalon M V, Cohen KR. Motivational Interviewing and Treatment Adherence among Psychiatric and Dually Diagnosed Patients. The Journal of Nervous \& Mental Disease. 1999; 187(10):630-5. doi: 10.1097/00005053-199910000-0000

[41] Green BL AM. Developing and Implementing a Programwide Vision for Effective Mental Health Consultation. Washington, D.C.: Center for Early Childhood Mental Health Consultation; 2012.

[42] Hansen DC, Matt-Hensrud N, Holland DE, Severson MA. Development of a discharge planning mentorship program. Journal for Nurses in Staff Development. 2000; 16(1):11-6. doi: 10.1097/00124645-200001000-00002

[43] Grant Jon E. Caring for your patient after discharge [Internet]. 2018 [Cited 2018 Feb 11]; Available from: https:/ / www.questia. com/library/journal/1G1-162867166/caring-for-your-patientafter-discharge

[44] National institute for Health and Care Excellence. Transition between inpatient mental health settings and community or care home settings [Internet]. 2016 [Cited 2018 Feb 19].Available from: https://www.nice.org.uk/guidance/ng53/evidence/fullguideline-pdf-2606951917
[45] Victoria's Hub for Health Services and Business. Discharge planning for adult community mental health services. Melbourne: Office of the Chief Psychiatrist; 2002. 